\title{
Health-care utilization in Dutch systemic sclerosis patients
}

\author{
Jessica Meijs • Elisabeth J. M. Zirkzee • Anne A. Schouffoer • Stella M. Henquet • \\ Monique A. A. Caljouw • Theo Stijnen • Tom W. J. Huizinga • \\ Annemie J. M. Schuerwegh • Theodora P. M. Vliet Vlieland
}

Received: 3 June 2013 / Revised: 16 July 2013 / Accepted: 13 August 2013

(C) Clinical Rheumatology 2013

\begin{abstract}
This study aims to examine healthcare utilization and its determinants among patients with systemic sclerosis (SSc). A cross-sectional survey among all patients with SSc visiting an outpatient clinic of an academic hospital in the Netherlands was done. Assessments included sociodemographic characteristics and a survey on healthcare utilization including a registration of contacts with healthcare services since onset of disease, contacts, and number of visits with healthcare services over the last 12 months. A total healthcare utilization score of all visits over the last 12 months was computed and classified as high and low care utilization according to the median. In addition, the Short Form-36 and the Scleroderma Health Assessment Questionnaire (SHAQ) were administered. Logistic regression analysis was used to determine the relationship between high and low healthcare utilization as
\end{abstract}

J. Meijs $(\varangle) \cdot$ E. J. M. Zirkzee • A. A. Schouffoer •

T. W. J. Huizinga $\cdot$ A. J. M. Schuerwegh

Department of Rheumatology, Leiden University Medical Center, P.O. Box 9600, 2300, RC Leiden, The Netherlands

e-mail: j.meijs@lumc.nl

A. A. Schouffoer

Department of Rheumatology, Haga Hospital,

The Hague, The Netherlands

S. M. Henquet

Department of Radiology, Leiden University Medical Center,

Leiden, The Netherlands

M. A. A. Caljouw

Department of Public Health and Primary Care, Leiden University

Medical Center, Leiden, The Netherlands

T. Stijnen

Department of Medical Statistics, Leiden University Medical Center, Leiden, The Netherlands

T. P. M. Vliet Vlieland

Department of Orthopaedics, Leiden University Medical Center, Leiden, The Netherlands dependent variable and sociodemographic and disease characteristics as independent variables. Sixty-four patients returned the questionnaires. Over the last 12 months, $83 \%$ of the patients had had contact with one or more physicians. On average, patients reported 3.9 visits $(\mathrm{SD}, 2.9)$ to a rheumatologist and 6.9 visits (SD, 9.3) to other medical specialists over the last 12 months. The median total health-care utilization was six visits over the last 12 months. Multivariate regression showed that a higher SHAQ score was significantly associated with higher healthcare utilization. Patients with SSc visited a considerable number of various health-care providers. Patients with more functional disability were using more healthcare.

Keywords Health-care utilization · Health-care surveys · Health services $\cdot$ Scleroderma $\cdot$ Systemic sclerosis

\section{Introduction}

Systemic sclerosis $(\mathrm{SSc})$ is a rare multisystem disease of unknown origin. It is a complex disease characterized by a triad of processes; vasculopathy, immune activation, and fibrosis [1]. SSc affects the skin and internal organs, resulting in a considerable discomfort, morbidity, and a decrease in health-related quality of life (HRQOL) [2]. Given the extensiveness of the disease, SSc patients usually require multidisciplinary treatment involving, apart from the rheumatologist, other medical specialists, and nonmedical health professionals such as physical therapists, occupational therapists, dieticians, nurses, or psychologists [3-5].

Research on the extent of health-care utilization among SSc patients is scarce. The available studies in general focused on cost of illness [6], sometimes combined with hospitalization rates [7, 8] or reported the utilization of health-care providers per year, without specifying the categories used for analysis or including all categories of health-care services [2, 3, 8-10]. Of these 
studies, most were performed in Canada and the USA, only one study was performed in Europe [9].

On average, the number of visits to the rheumatologist varied from 2.4 until 4.0 visits per year $[3,9,11]$, to other medical specialists from 3.8 until 7.1 visits per year [3, 10], and to the general practitioner from 3.5 until 10.3 visits per year $[3,8,11]$. Regarding the utilization of care provided by health professionals other than physicians, in the study by Johnson et al. [4], 31-46\% of the patients had visited the physiotherapist and 16-34\% of the patients had visited the occupational therapist over a period of 1 year. Patient and disease characteristics that explain the use of this health care are lacking. Only one of these studies focused on factors associated with health-care utilization. In that study from Canada, where access to health care is readily available and free of costs, it was found that higher skin scores, more comorbidity, and worse physical functioning were associated with higher health-care utilization [3].

A number of studies have investigated the unmet needs and preferences regarding health-care delivery among patients with SSc, concluding that unmet health care and information needs are common $[3,12,13]$.

For improving health care in patients with SSc, it is important to know which kind of patients are at risk having a high health-care utilization. There is a lack of information about this topic, especially in Europe. Therefore, the aim of this study was to examine the extent of the use of all categories of healthcare and its determinants among Dutch patients with SSc.

\section{Patients and methods}

\section{Study design}

The study had a cross-sectional design. Ethical approval for this study was obtained from the Institutional Review Board of the Leiden University Medical Center. All participants gave written informed consent. This study was part of a larger project, also including assessment of health care and information needs among patients with SSc [5].

\section{Patients}

Patients were recruited between May and July 2007. Inclusion criteria were diagnosis of SSc as established by their treating rheumatologist according to American College of Rheumatology criteria [14], having visited the rheumatologist at the outpatient clinic Leiden University Medical Center less than 24 months ago, being able to comprehend a Dutch questionnaire, and age 18 years or older. A questionnaire was sent to all patients meeting these criteria, accompanied by an invitation letter, an information leaflet explaining the aim and the methods of the study, and a consent form. A follow-up letter was sent to participants who did not return the surveys within a month.

Assessment methods

\section{Survey on health-care utilization}

The survey on health-care usage was based on a questionnaire employed in a previous study among patients with rheumatoid arthritis [15]. The survey included the following categories of health-care services (number of different health-care professionals/services within a category): (1) rheumatologist, (2) medical specialists other than rheumatologist, (3) general practitioner, (4) dental care, (5) health professionals, (6) home care, (7) hospital admission, and (8) day-patient care. The category medical specialists included internist, nephrologist, pulmonologist, cardiologist, dermatologist, neurologist, psychiatrist, ear nose and throat specialist, and (plastic) surgeon. Dental care included the dentist and dental hygienist. Health professionals included physiotherapist, occupational therapist, nurse specialist, dietician, social worker, and psychologist. Care at home included home help and community nurse.

With every health-care provider or service, patients were asked "Did you have contact with a... related to SSc since the onset of your disease/over the past 12 months?" (yes/no). If the answer was "yes" with respect to the past 12 months, patients were asked how many contacts or treatment days they had received in total. For each category, the number of contacts and visits within the last 12 months were counted, including patients having one or more health-care providers per category visited.

Exceptions were treatment by the physiotherapist and home help with usually either none or more than 12 contacts per year (physiotherapist ranged 0-96; home help ranged 0792 visits per year), where it was asked how many times per week patients had received this kind of health care on average. A total health-care utilization score of all visits in the last 12 months was computed, excluding rheumatologist care as only patients who had visited the rheumatologist at least once in the past 24 months were included in this study. Health-care utilization was classified as high care utilization and low care utilization according to the median of the total number of visits in the last year, including patients with the exact number of the median total health-care utilization score in the high care utilization category.

\section{Sociodemographic characteristics}

Sociodemographic variables included age, status of living (living with a partner yes/no), educational level: primary education ( $0-8$ years; low education level), secondary education (9-16 years; medium education level) and higher 
vocational education/ university (post secondary; high education level), and paid employment (yes/no).

\section{Disease characteristics and function}

Disease duration (years) and disease subset (limited or diffuse) [14] were derived from the medical record. In addition, patients were asked to fill in the Scleroderma Health Assessment Questionnaire (SHAQ) - a 20-item questionnaire comprising eight domains of activities of daily living, with the final score ranging from 0 (no disability) to 3 (severe disability) with scleroderma symptom visual analog scales $(0-100 \mathrm{~mm})$, Raynaud's disease, digital ulcers, intestinal complaints, pulmonary complaints, overall complaints, and pain [16]. The SHAQ has been found to be a reliable outcome measure for disease severity in SSc [17].

The presence of the autoantibodies antinuclear antibody (ANA), antitopoisomerase I (anti-Scl70), anticentromere, and anti-RNP and the laboratory values erythrocyte sedimentation rate, $\mathrm{C}$-reactive protein, and creatinine as measured at the visit closest to filling out the questionnaire were noted. In addition, interstitial lung disease (ILD), vital capacity (VC), and the presence of pulmonary hypertension were derived from the medical records.

\section{Quality of life}

Quality of life was measured with the Short Form-36 (SF-36), which contains eight subscales: physical functioning, role limitation due to physical problems, bodily pain, general health perception, vitality, social functioning, role limitation due to emotional problems, and mental health. The scoring range of the SF-36 subscales is [0-100], with higher scores indicating better quality of life. The subscales can be converted into two summary scales: the physical component summary (PCS) and mental component summary (MCS) scale, standardized to a score with a mean of 50 and a standard deviation of 10 in the general population. For that purpose, we used the scores from an age- and sex-matched, normative sample, drawn from a large, random, nationwide sample of adults $(n=1,742)$ from the general Dutch Population Frequency Table and factor score coefficients [18]. The psychometric properties of this questionnaire have been found to be adequate [19].

\section{Data analysis}

According to their distribution, continuous variables were either presented as mean and standard deviation (SD) or medians with interquartile range (p25-p75). All continuous variables were compared between patients with diffuse $\mathrm{SSc}$ and limited SSc by the Mann-Whitney $U$ test.

Categorical variables were calculated as frequencies with percentages and compared between patients with diffuse and limited SSc by using Chi-square analyses and Fisher's exact test where applicable. Use of health care was calculated per provider or service and presented as absolute number of patients using that provider or service and by the mean (SD) number of visits per patient (total health-care utilization score).

Univariate logistic regression analysis was used to determine the relationship between health-care utilization as dependent factor (high or low health-care utilization score) and all possible contributing factors (sociodemographic and disease characteristics). When needed, due to cells with zero patients with high or low healthcare utilization score, exact logistic regression was applied. Associations were expressed as odds ratios (ORs) with the $95 \%$ confidence intervals (CIs) and $p$ value. Possible correlations between variables which were significantly contributing in the univariate logistic regression analysis were also examined to check for multicollinearity before inclusion, with variance inflation factor (VIF) of $<10$. A VIF of 10 indicates that (all other things being equal) the variance of the $i$ th regression coefficient is 10 times greater than it would have been if the $i$ th independent variable had been linearly independent of the other independent variable in the analysis [20]. In case of multicollinearity between variables, the most significant variable will be included in the multivariate analysis.

All variables with a $p<0.10$ in the univariate logistic regression analysis were then included in a multivariate logistic regression analysis using the backward method in order to evaluate their independent association with health-care usage. The multivariate analysis was adjusted for sex, age, and disease duration. For all analyses, results were considered statistically significant if the $p$ value was smaller than 0.05 (two-sided).

Data entry was performed using Microsoft Office Access 2003. All statistical analyses were executed using SPSS 17.0 software (SPSS Inc., Chicago, USA), except for exact logistic regression which was done using SAS 9.2 software (SAS Institute Inc., Cary, USA).

\section{Results}

Patient population

Sociodemographic and disease characteristics are presented in the article of Schouffoer et al. [5, Table 1]. Briefly, 64 patients returned the questionnaire, including 34 patients with diffuse SSc.

Description of use of care

The use of health-care services by patients is shown in Table 1.

Physicians

Over the last 12 months, $83 \%$ of the patients had had contact with one or more physicians, with the median number of contacts 
Table 1 Health-care utilization of 64 patients with systemic sclerosis
ENT ear nose and throat, $N$ number, $S D$ standard deviation ${ }^{\mathrm{a}}$ Visits/week

\begin{tabular}{|c|c|c|c|}
\hline Health-care service & $\begin{array}{l}\text { Contact since } \\
\text { Onset of disease, } N(\%)\end{array}$ & $\begin{array}{l}\text { Contact in last } \\
12 \text { months, } N(\%)\end{array}$ & $\begin{array}{l}\text { Number of visits per patient in last } \\
12 \text { months, if at least one, mean (SD) }\end{array}$ \\
\hline Rheumatologist & $64(100)$ & $48(75)$ & $3.9(2.9)$ \\
\hline Medical specialists & $58(90.6)$ & $40(62.5)$ & $6.9(9.3)$ \\
\hline Internist & $26(40.6)$ & $9(14.1)$ & $2.3(1.8)$ \\
\hline Nephrologist & $10(15.6)$ & $4(6.3)$ & $4.5(5.0)$ \\
\hline Pulmonologist & $39(60.9)$ & $18(28.1)$ & $3.0(2.2)$ \\
\hline Cardiologist & $37(57.8)$ & $21(32.8$ & $1.9(1.2)$ \\
\hline Dermatologist & $38(59.4)$ & $16(25)$ & $5.9(10.7)$ \\
\hline Neurologist & $11(17.2)$ & $5(7.8)$ & $4.5(5.2)$ \\
\hline Psychiatrist & $4(6.3)$ & $2(3.1)$ & $6.5(2.1)$ \\
\hline ENT specialist & $15(23.4)$ & $6(9.4)$ & $1.4(0.5)$ \\
\hline (Plastic) surgeon & $10(15.6)$ & $4(6.3)$ & $2.0(0.0)$ \\
\hline General practitioner & $56(87.5)$ & $20(31.3)$ & $3.8(2.9)$ \\
\hline Dental care & $33(51.6)$ & $20(31.3)$ & $3(1.5)$ \\
\hline Dentist & $31(48.4)$ & $16(25)$ & $2.2(0.8)$ \\
\hline Dental hygienist & $19(29.7)$ & $13(20.3)$ & $1.9(1.0)$ \\
\hline Health professionals & $55(85.9)$ & $39(60.9)$ & $3.9(4.6)$ \\
\hline Physiotherapist & $52(81.3)$ & $37(57.5)$ & $1.3(0.5)^{\mathrm{a}}$ \\
\hline $\begin{array}{l}\text { Occupational } \\
\text { therapist }\end{array}$ & $9(1.41)$ & $9(14.1)$ & $2.1(1.1)$ \\
\hline Nurse specialist & $27(42.4)$ & $8(12.5)$ & $3.5(3.5)$ \\
\hline Dietician & $19(29.7)$ & $11(17.2)$ & $2.3(1.4)$ \\
\hline Social worker & $24(37.5)$ & $13(20.3)$ & $2.7(1.5)$ \\
\hline Psychologist & $5(7.8)$ & $2(3.1)$ & $1.5(0.7)$ \\
\hline Care at home & $15(23.4)$ & $10(15.6)$ & $8.4(16.4)$ \\
\hline Home help & $12(18.8)$ & $8(12.5)$ & $1.9(1.0)^{\mathrm{a}}$ \\
\hline Community nurse & $14(21.9)$ & $4(6.3)$ & $17.3(23.4)$ \\
\hline Hospital admission & $60(93.8)$ & $9(14.1)$ & $5(5.3)$ \\
\hline Day patient care & $32(50)$ & $18(28.1)$ & $2.1(2.9)$ \\
\hline
\end{tabular}

being 4 (range, 0-14). Regarding this physicians visits, the rheumatologist, cardiologist, general practitioner, pulmonologist, and dermatologist were most frequently mentioned, in this order.

On average, patients reported 3.9 visits (SD, 2.9) to a rheumatologist, 6.9 visits (SD, 9.3) to other medical specialists, and 3.8 (SD, 2.9) to the general practitioner in the last 12 months, if they had had at least one visit.

Dental care

Over the last 12 months, $25 \%$ of the patients had had contact with the dentist and $20 \%$ had had contact with the dental hygienist.

Health professionals

Sixty-one percent of the patients had had contact with one or more health professionals in the last 12 months, with the physiotherapist being most frequently mentioned. On average, patients visited the physiotherapist 1.3 (SD, 0.5) times per week.

Home care

Over the last 12 months, $16 \%$ of the patients had had care at home, most frequently receiving home help, with a mean of 1.9 (SD 1.0) visits per week.

Health admission and day-patient care

Fourteen percent of the patients were admitted to a hospital over the last 12 months, in case of admission the frequency was on average five admissions in the last year. Over the last 12 months, $28 \%$ of the patients had had contact with our daypatient care, with a mean of 2.1 (SD, 2.9) visits. 
Determinants of health-care utilization

The median total health-care utilization was 6 (range, 0-62) visits over the last 12 months. Table 2 shows univariate and exact multivariate logistic regression analysis of sociodemographic and disease characteristics of high and low care users as classified by median of total health-care utilization scores in the last 12 months. The univariate logistic regression analysis showed that higher SHAQ total score (OR, 3.344; $95 \%$ CI, 1.335-8.252), low PCS scores (OR, 0.948; $95 \%$ CI, 0.902-0.997) and anti-Scl70 were significantly associated with high health-care utilization. Living without a partner, low MCS score, higher educational level and ANA were associated with a higher health-care utilization $(p$ value

Table 2 Characteristics of patients with systemic sclerosis divided into high and low total health-care utilization over 12 months

\begin{tabular}{|c|c|c|c|c|c|c|}
\hline & $\operatorname{High} N=34$ & Low $N=30$ & $\begin{array}{l}\text { Univariate odds } \\
\text { ratio }(95 \% \mathrm{CI})\end{array}$ & $p$ value & $\begin{array}{l}\text { Multivariate } \\
\text { odds ratio }(95 \% \mathrm{CI})\end{array}$ & $p$ value \\
\hline Diagnosis: DcSSC, $N(\%)$ & $20(58.8)$ & $14(46.7)$ & $1.633(0.606-4.396)$ & 0.332 & & \\
\hline Age, years; mean (SD) & $56.7(13.3)$ & $53.7(13.4)$ & $1.017(0.979-1.057)$ & 0.375 & & \\
\hline Female, $N(\%)$ & $26(76.5)$ & $24(80)$ & $1.231(0.373-4.066)$ & 0.733 & & \\
\hline Disease duration, years; mean (SD) & $7.5(6.0)$ & $9.1(6.7)$ & $0.960(0.887-1.040)$ & 0.318 & $0.898(0.782-1.031)$ & 0.127 \\
\hline Caucasian origin; $N(\%)^{\mathrm{a}}$ & $31(91.2)$ & $28(93.3)$ & $1.355(0.211-8.710)$ & 0.749 & & \\
\hline Living with partner, $N(\%)$ & $21(61.8)$ & $25(83.3)$ & $3.095(0.948-10.107)$ & 0.061 & & \\
\hline Paid employment, $N(\%)^{\mathrm{a}}$ & $13(38.2)$ & $9(30)$ & $0.659(0.231-1.880)$ & 0.436 & & \\
\hline \multicolumn{7}{|l|}{ Education level, $N(\%)^{\mathrm{a}}$} \\
\hline Low & $14(41.2)$ & $12(40)$ & $0.438(0.094-2.030)$ & 0.291 & & \\
\hline Medium & $10(29.4)$ & $14(46.7)$ & $0.268(0.057-1.269)$ & 0.097 & & \\
\hline High & $8(23.5)$ & $3(10)$ & Reference cat. & & & \\
\hline \multicolumn{7}{|l|}{ Autoantibodies, $N(\%)^{\mathrm{a}}$} \\
\hline ANA & $32(94.1)$ & $23(76.7)$ & $17.979(0.965-335.035)$ & 0.053 & & \\
\hline Anti-Scl70 & $2(5.9)$ & $10(33.3)$ & $0.160(0.032-0.801)$ & 0.025 & & \\
\hline Anti-centromere & $5(14.7)$ & $4(13.3)$ & $1.511(0.365-6.260)$ & 0.570 & & \\
\hline Anti-RNP & $1(2.9)$ & $4(13.3)$ & $0.219(0.027-2.114)$ & 0.189 & & \\
\hline $\mathrm{ESR}, \mathrm{mm} ;$ mean $(\mathrm{SD})^{\mathrm{a}}$ & $22.2(14.9)$ & $25(17.2)$ & $0.989(0.958-1.021)$ & 0.491 & & \\
\hline $\mathrm{CRP}, \mathrm{mg} / \mathrm{dl}$, mean $(\mathrm{SD})^{\mathrm{b}}$ & $7.6(9.1)$ & $5.4(3.5)$ & $1 / 063(0.953-1.187)$ & 0.272 & & \\
\hline Creatinin, $\mu \mathrm{mol} / 1$, mean $(\mathrm{SD})^{\mathrm{a}}$ & $81(40.6)$ & $75.3(24.5)$ & $1.005(0.990-1.021)$ & 0.512 & & \\
\hline Interstitial lung disease, $N(\%)^{\mathrm{a}}$ & $12(35.5)$ & $12(40)$ & $0.850(0.304-2.378)$ & 0.757 & & \\
\hline Vital capacity, $\%$ expected; mean $(\mathrm{SD})^{\mathrm{c}}$ & $92.1(18.8)$ & $91.9(20.1)$ & $1.001(0.974-1.029)$ & 0.955 & & \\
\hline Pulmonary hypertension, $N(\%)^{\mathrm{a}}$ & $2(5.9)$ & 0 & $2.028(0.155-\infty)$ & 0.497 & & \\
\hline $\begin{array}{l}\text { SHAQ }(0-3) \text {; median } \\
\quad(25-75 \text { th percentile })^{\mathrm{a}}\end{array}$ & $0.9(0.3-1.7)$ & $0.4(0.1-0.9)$ & $3.344(1.355-8.252)$ & 0.009 & $4.721(1.364-16.345)$ & 0.014 \\
\hline VAS Raynaud's disease (0-100) & $31(12-52)$ & $25(13.5-65.5)$ & $0.998(0.981-1.015)$ & 0.810 & & \\
\hline VAS digital ulcers $(0-100)$ & $13.5(0-40)$ & $0(0-40)$ & $0.997(0.981-1.013)$ & 0.712 & & \\
\hline VAS intestinal complaints $(0-100)$ & $13(0-41.8)$ & $14(1-49.5)$ & $1.000(0.983-1.018)$ & 0.981 & & \\
\hline VAS pulmonary complaints $(0-100)$ & $18.5(0-610$ & $20(1-50.5)$ & $1.003(0.986-1.020)$ & 0.757 & & \\
\hline VAS overall complaints $(0-100)$ & $25.5(10-65)$ & $31(8-50)$ & $1.005(0.986-1.025)$ & 0.583 & & \\
\hline VAS pain $(0-100)$ & $27(6.5-53)$ & $19(1.5-52)$ & $1.005(0.988-1.022)$ & 0.572 & & \\
\hline \multicolumn{7}{|c|}{ SF-36 $(0-100)$; median $\left(25-75{\text { th percentile })^{c}}^{c}\right.$} \\
\hline $\begin{array}{l}\text { Physical Component Summary } \\
\text { Scale }(0-100)\end{array}$ & $39.7(31.5-48.8)$ & $47.1(37.8-57.6)$ & $0.948(0.902-0.997)$ & 0.037 & & \\
\hline $\begin{array}{l}\text { Mental Component Summary } \\
\text { Scale }(0-100)\end{array}$ & $54.5(38.3-61.1)$ & $58.1(49.4-63.6)$ & $0.951(0.903-1.002)$ & 0.059 & & \\
\hline
\end{tabular}

$N$ number, $C I$ confidence interval, DcSSC diffuse cutaneous systemic sclerosis, $S D$ standard deviation, SHAQ Scleroderma Health Assessment Questionnaire, VAS visual analog scale, $S F-36$ Short Form-36

${ }^{\mathrm{a}}<5 \%$ missing

${ }^{\mathrm{b}}<10 \%$ missing

${ }^{c}<15 \%$ missing 
$<0.10)$. Since ANA had a widespread $95 \%$ CI $(0.965-$ 335.035), ANA was not included in the multivariate analysis.

In the final multivariate analysis only higher SHAQ total score (OR, 3.344; 95 \% CI, 1.335-8.252) was found to be significantly associated with higher health-care utilization. This result did change if the model included correction for sex, age, and disease duration. Shorter disease duration (OR, 0.898; $95 \%$ CI, 0.782-1.031) was associated with higher health-care utilization, although this was not significant. Additionally, in order to achieve information about patients visiting the most obvious health-care providers, the number of visits to the pulmonologist and cardiologist were analyzed for patients with serious cardiology and pulmonology involvement, respectively, suffering from pulmonary hypertension and ILD and VC below $70 \%$ of predicted [21]. The main finding was that patients with pulmonary hypertension properly visited both medical specialists. In contrast, only $35 \%$ of the patients with ILD were visiting the pulmonologist (data not shown) although $86 \%$ of the patients with a decreased VC below $70 \%$ of predicted had visited the pulmonologist.

\section{Discussion}

In this cross-sectional study among Dutch SSc patients, health-care utilization was found to be substantial and included all categories of health care. A higher SHAQ score was significantly associated with more health-care utilization, implying that patients with a higher disease severity have higher health-care utilization. Patients with lower physical quality of life and positive autoantibodies were more likely to use more health care, although this was not significant in the multivariate analysis.

Our results are largely in line with the available literature reporting health-care utilization over a period of 12 months [3, 4, 9-11], despite differences in the patient characteristics. The populations of those studies are comparable with our population in respect to age, disease duration, and origin. But our study comprised relatively more male patients than those studies, where the male patients varied between 10 and $14 \%[3,4,9-11]$ of the patients.

With respect to the frequency of contacts with health-care providers per year, the number of outpatient visits to the rheumatologist and other medical specialists was comparable to the findings of previous research [3, 9-11]. In our study, $75 \%$ of the patients had had visited the rheumatologist over the last 12 months, similar to other studies where $69-90 \%$ of the patients visited the rheumatologist over the last year [4]. Visits to other medical specialists were comparable to the previous studies $[3,10]$, but still not as frequent as would be expected in order to the presence of disease involvement.

Regarding visits to the general practitioner over the past year, in previous studies the rates ranged between 76 and $81 \%$ of the patients $[3,4,10]$. In our study, $31 \%$ of the patients had had visited the general practitioner, with a mean of 3.8 visits in the last year. In contrast, the number of visits to the general practitioner was 10.3 in the Hungarian study [10].

One of the possible explanations can be that in our study, we explicitly asked patients to report only visits related to their SSc. Another reason can be the differences in distances to the hospital. The distance between the patients' place of living and the hospital is relatively long in Hungary. Moreover, the public transport network of Hungary is not comparable with the Dutch public network.

The number of visits regarding the occupational therapist in our study was comparable to the study by Johnson et al. [4], whereas comparisons regarding physical therapy are difficult to make due to differences in the measurement method.

The frequencies of visits to dental care services seemed to be lower in our patients compared to the general population of the Netherlands. In the study of Listl et al. [22], 65.7\% of the respondents aged 50+ had visited dental care services in the last 12 months, in contrast to our patients of whom $25 \%$ visited dental care the last 12 months and $48.4 \%$ since onset of disease.

Regarding the determinants of health-care utilization, only one study is comparable to ours [3]. In that study, high healthcare utilization was defined as a median of six physician visits over the last year, similar to our study where a median total number of visits of six or more per year were used.

With respect to the relation of disease characteristics and health-care utilization, Bernatski et al. found in multivariate logistic regression analysis more physician visits among patients with diffuse SSc and identified worse physical functioning, higher skin scores, and more comorbidity as predictors [3]. In our study, a high SHAQ score was significantly associated with health care. A similar finding was seen in patients with other rheumatologic diseases. For instance, in patients with SLE, a high HAQ score is significantly associated with health-care utilization [23]. This study also established a significant association of younger age, major organ involvement and the use of immunosuppressive medication on high healthcare utilization. In patients with rheumatoid arthritis, high health-care utilization was related to age, sex, living situation, and disease-related factors [15].

In line with Bernatski et al., we did not found an association with pulmonary arterial hypertension and lung involvement and health-care utilization. Bernatski et al. suggested that pulmonary hypertension and lung involvement were not independently associated as these conditions are usually related to overall organ involvement. In our study, laboratory markers were not associated with health-care utilization. The presence of autoantibodies was significantly associated with high health-care utilization in univariate logistic regression analysis, but in multivariate analysis no relation was observed. This is probably due to the inter-relationship between the presence 
of autoantibodies and other factors associated with health-care usage, such as worse physical functioning. It is known from literature that there is a correlation of demographic characteristics and symptoms of SSc with the presence (or absence) of autoantibodies [24]. To the best of our knowledge, nothing is known about the relationship of autoantibodies and SHAQ scores in patients with SSc.

With respect to the sociodemographic characteristics and health-care utilization, Bernatski et al. found a higher income being associated with health-care utilization. In our study, none of the sociodemographic variables were associated. Comparison with other patient groups may be hampered by differences in healthcare systems and socioeconomic background of patients. The study of Bernatski et al. [3] was performed in Canada, where all citizens and landed immigrants have access to primary care, specialist care, and medically necessary investigations, free of cost to the patient [4].

This study has several limitations which should be taken into account. First, a selection bias cannot be fully excluded. Our cohort consisted of a selection of patients who were treated by a rheumatologist in a university hospital. The patient population may however be comparable to SSc patients under the care of rheumatologists in general hospitals, as our hospital is the only hospital offering rheumatology services in the Leiden region.

Second, the study group is small, however the response rate of $83 \%$ is high, and therefore this study is a good representation of the patients attending our clinic.

Third, our SSc patients demonstrated a relatively mild functional disability in both the diffuse and limited group as compared to cohorts in other studies published on Quality of Life and Health Care issues in SSc $[2,18]$.

Fourth, it was not possible to assess the disease severity score according to Medsger et al. [25], since not all clinical parameters, for instance modified Rodnan Skin Score and gastro-intestinal involvement, as well as visits to the gastroenterologist, were consistently recorded in all the patients. Based on the low prevalence of pulmonary hypertension and lung involvement in our patients, we concluded our patients have a relative mild disease [26].

Fifth, the amount of health-care utilization is a patientreported outcome potentially leading to recall bias. Unfortunately it was not possible to validate the number of visits reported by patients themselves on the basis of visit registration, since most health-care providers were providing health care in other hospitals or in primary care.

Furthermore, health-care utilization was counted as a total score of all visits in the last 12 months, with the exception of physiotherapist and home help, where it was asked how many times per week patients had received this kind of health care. We counted these visits per week instead of per year, because of the wide range of number of visits per year. This is due to some patients needed home care three times a day, which will be scattering the median health-care utilization to unequal heights. We consider this dependent to the kind of these forms of health delivery.

Finally, specific reference patterns were not recorded. It is unknown whether health-care utilization was due to SSc or not. Besides that, emergency room visits were not taken into account separately. In case of emergencies related to their disease, patients usually come to the outpatient clinic and not to the emergency room. If emergency room visits had been counted, the rates would most probably be lower.

In a previous study in SSc patients on preferences for health-care delivery, a yearly standardized multidisciplinary assessment in a day program was found to be highly preferred [5]. Probably this is explained by the complexity of healthcare utilization in SSc, in which communication between physicians is of utmost importance and multiple visits to the hospital place a high burden on patients.

To the best of our knowledge, this is the first study on health-care utilization by patients with SSc and the patient and disease characteristics that explain the use of this health care in (the Western part of) Europe. SSc patients visit various healthcare providers emphasizing the complexity of health-care delivery in this disease. Patients with a higher burden of disease are using more health care, although it was not that frequent as would be expected in order to the presence of disease involvement. A standardized multidisciplinary assessment is preferred by the SSc patients.

Acknowledgments This study was funded by nonprofit foundation National Reumafonds (Dutch Arthritis Foundation). Jessica Meijs was supported by an unrestricted educational grant of Actelion Pharmaceuticals Nederland BV (Woerden, The Netherlands).

\section{References}

1. Charles C, Clements P, Furst DE (2006) Systemic sclerosis: hypothesis-driven treatment strategies. Lancet 367:1683-1691

2. Hudson M, Thombs BD, Steele R, Panopalis P, Newton E, Baron M (2009) Quality of life in patients with systemic sclerosis compared to the general population and patients with other chronic conditions. J Rheumatol 36:768-772

3. Bernatsky S, Panopolis P, Hudson M, Pope J, LeClercq S, Robinson $\mathrm{R}$ et al (2009) Demographic and clinical factors associated with physician services use in systemic sclerosis. J Rheumatol 36:96-98

4. Johnson SR, Carette S, Dunne JV (2006) Scleroderma: health services utilization from patients' perspective. J Rheumatol 33:1123-1127

5. Schouffoer AA, Zirkzee EJM, Henquet SM, Caljouw MAA, SteupBeekman GM, Laar JMV et al (2011) Needs and preferences regarding health care delivery as perceived by patients with systemic sclerosis. Clin Rheumatol 30:815-824

6. Bernatsky S, Hudson M, Panopalis P, Clarke AE, Pope J, Leclercq S et al (2009) The cost of systemic sclerosis. Arthritis Rheum 61:119-123

7. Chung L, Krishnan E, Chakravarty EF (2007) Hospitalizations and mortality in systemic sclerosis: results from the Nationwide Inpatient Sample. Rheumatol 46:1808-1813 
8. Nietert PJ, Silverstein MD, Silver RM (2001) Hospital admissions, length of stay, charges, and in-hospital death among patients with systemic sclerosis. J Rheumatol 28:2031-2037

9. Wilson L (1997) Cost-of-illness of scleroderma: the case for rare diseases. Arthritis Rheum 27:73-84

10. Minier T, Pentek M, Brodszky V, Ecseki A, Karpati K, Polgar A et al (2010) Cost-of-illness of patients with systemic sclerosis in a tertiary care centre. Rheumatol 49:1920-1928

11. Furst DE, Fernandes AW, Iorga SR, Greth W, Bancroft T (2012) Annual medical costs and healthcare resource use in patients with systemic sclerosis in an insured population. J Rheumatol 39:23032309

12. Rubenzik TT, Derk CT (2009) Unmet patient needs in systemic sclerosis. J Clin Rheumtol 15:106-110

13. Van der Vaart R, Repping-Wuts H, Drossaert CHC, Taal E, KnaapenHans HKA, Van de Laar MAFJ (2013) Needs for online information and support of patients with systemic sclerosis. Arthritis Care Res 65: 594-600

14. Preliminary criteria for the classification of systemic sclerosis (scleroderma). Subcommittee for Scleroderma. Criteria of the American Rheumatism Association Diagnostic and Therapeutic Criteria Committee. Arthritis Rheum 1980;23:581-590

15. Jacobi CE, Triemstra M, Rupp I, Dinant HJ, Van den Bos GAM (2001) Health care utilization among rheumatoid arthritis patients referred to a rheumatology center: unequal needs, unequal care? Arthritis Care Res 45:324-330

16. Clements PJ, Wong WK, Hurwitz EL, Furst DE, Mayes M, White B et al (2001) The disability index of the health assessment questionnaire is a predictor and correlate of outcome in the high-dose versus low-dose penicillamine in systemic sclerosis trail. Arthritis Rheum 44:653-661

17. Merkel PA, Herlyn K, Martin RW, Anderson JJ, Mayes MD, Bell P et al (2002) Measuring disease activity and functional status in patients with scleroderma and Raynaud's phenomenon. Arthritis Rheum 46:2410-2420

18. Ware JJ, Snow K, Kosinki M (1994) SF-36 health survey: manual and interpretation guide. The Health Institute, New England Medical Center, Boston, MA

19. Essink-Bot ML, Krabbe PF, Bonsel GJ, Aaronson NK (1997) An empirical comparison of four generic health status measures. The Nottingham Health Profile, the medical outcome study 36-item Short-Form health survey, the COOP/WONCA charts, and the EuroQol Instrument. Med Care 35:522-537

20. O'Brien RM (2007) A caution regarding rules of thumb for variance inflation factors. Qual Quant 41:673-690

21. Goh NSL, Desai SR, Veeraraghavan S, Hansell DM, Copley S et al (2008) Interstitial lung disease in systemic sclerosis. Am J Respir Crit Care Med 177:1248-1254

22. Listl S, Moran V, Maurer J, Faggion CM Jr (2012) Dental service utilization by Europeans aged 50 plus. Community Dent Oral Epidemiol 20:164-174

23. Zirkzee EJM, Steup-Beekman GM, Schouffoer AA, Henquet SM, Caljouw MAA, Huizinga TWJ et al (2011) Health care usage in Dutch systemic lupus erythematosus patients. Lupus 20:1147-1154

24. Mierau R, Moinzadeh P, Riemekasten G, Melchers I, Meurer M, Reichenberger $\mathrm{F}$ et al (2011) Frequency of disease-associated and other nuclear autoantibodies in patients of the German network for systemic scleroderma: correlation with characteristic clinical features. Arthritis Res Ther 13:R172

25. Medsger TA Jr, Bombardieri J, Czirjak L, Scorza R, Della Rossa A, Bencivelli W (2003) Assessment of disease severity and prognosis. Clin Exp Rheumatol 21(suppl 29):S42-S46

26. Nihtyanova SI, Tang EC, Coghlan JG, Wells AU, Black CM, Denton CP (2010) Improved survival in systemic sclerosis is associated with better ascertainment of internal organ disease: a retrospective cohort study. Q J Med 103:109-115 